\title{
The Connection between an Euclidean Gauss Markov Vector Field and the Real Proca Wightman Field
}

\author{
Te Hai Yao \\ Mathematics Department, Bedford College, London, England \\ Received August 12, 1974; in revised form October 16, 1974
}

\begin{abstract}
We construct an Euclidean Gauss Markov Vector Field which leads to the Real Proca Wightman Field describing particles of mass $>0$ and spin 1, for space-time dimension equal to 4 .
\end{abstract}

\section{The Result}

We have constructed a vector random field $\Phi(f)=\left\{\phi_{0}(f), \varphi_{1}(f), \varphi_{2}(f), \varphi_{3}(f)\right\}$ on a probability space $(\Omega, \mathscr{B}, \mu)$ over $\mathscr{D}_{r}\left(R^{4}\right)$, the space of real $C^{\infty}$ functions with compact support in $R^{4}$, the field being Gaussian, Euclidean and Markov in a sense defined below. We call the field $\hat{\Phi}(f)=\left\{\hat{\varphi}_{0}(f), \hat{\varphi}_{1}(f), \hat{\varphi}_{2}(f), \hat{\varphi}_{3}(f)\right\}$ $=\left\{-i \phi_{0}(f), \varphi_{1}(f), \varphi_{2}(f), \varphi_{3}(f)\right\}$ a Schwinger field. Then the general covariance $E \hat{\varphi}_{\mu}(f) \hat{\varphi}_{v}(g)$ of the Schwinger field are given by

$$
\iint d x d y f(x) g(y) S_{\mu v}(x-y)
$$

where

$$
S_{\mu \nu}(x-y)=W_{\mu \nu}\left(-i\left(x_{0}-y_{0}\right),\left(x_{1}-y_{1}\right),\left(x_{2}-y_{2}\right),\left(x_{3}-y_{3}\right)\right)
$$

is the two point Schwinger function of the quantum theory of a free real vector Wightman field in 4-dimensional space time of mass $m>0$ satisfying the subsidiary condition $\partial_{0} \theta_{0}(x)=\sum_{\mu=1}^{3} \partial_{\mu} \theta_{\mu}(x)$ and describing particles of mass $m$ and spin 1 , i.e. the Real Proca Wightman Field, $W_{\mu \nu}(x-y)$ being the corresponding two point Wightman function $\left(\Psi_{0}, \theta_{\mu}(x) \theta_{v}(y) \Psi_{0}\right)$ where $\Psi_{0}$ is the vacuum of the Wightman theory and $\theta_{\mu}(x), \theta_{v}(y)$ are the corresponding quantum fields. Further, the expectation value

$$
\begin{aligned}
& E \hat{\phi}_{\mu_{1}}\left(f_{1}\right) \ldots \hat{\phi}_{\mu_{n}}\left(f_{n}\right) \\
&=\int \cdots \int d x^{(1)} \ldots d x^{(n)} f_{1}\left(x^{(1)}\right) \ldots f_{n}\left(x^{(n)}\right) S_{\mu_{1} \ldots \mu_{n}}\left(x^{(1)}, \ldots x^{(n)}\right) \\
& x^{(i)} \in R^{4}
\end{aligned}
$$

$S_{\mu_{1} \ldots \mu_{n}}\left(x^{(1)} \ldots x^{(n)}\right)$ being the $n$-point Schwinger function. We may extend the field over $\mathscr{D}_{r}\left(R^{4}\right)$ to an Euclidean Markov field over $\mathscr{D}\left(R^{4}\right)$, the space of $C^{\infty}$ functions with compact support in $R^{4}$, by linearity. We mention in passing that the statement $\Sigma_{\mu=0}^{3} \varphi_{\mu}\left(\partial_{\mu} f\right)=0$ for all $f \in \mathscr{D}_{r}\left(R^{4}\right)$ is not valid. We would like to mention that L. Gross [1] has also studied an Euclidean Proca Field. 


\section{Definitions and the Real Proca Wightman Field}

A Gaussian vector random field over $\mathscr{D}_{r}\left(R^{4}\right)$ is a collection of vector-valued random variables $\Phi(f)=\left\{\varphi_{0}(f), \varphi_{1}(f), \varphi_{2}(f), \varphi_{3}(f)\right\}$ on a probability space $(\Omega, \mathscr{B}, \mu)$ indexed by functions belonging to $\mathscr{D}_{r}\left(R^{4}\right)$ such that any finite collection of the components of the vector random variable(s) form a set of jointly Gaussian random variables and such that $\varphi_{\mu}(f)$ is linear and such that $\varphi_{\mu}\left(f_{\alpha}\right) \rightarrow \varphi_{\mu}(f)$ in measure if $f_{\alpha} \rightarrow f$ in the usual topology of $\mathscr{D}_{r}\left(R^{4}\right)$. We now let $\mathscr{A}=(a, A)$ be an element of the full Euclidean group on $R^{4}, A$ being a rotation and $a$ a translation, then a vector random field over $\mathscr{D}_{r}\left(R^{4}\right)$ is said to be Euclidean if

where

$$
E \varphi_{\mu_{1}}\left(f_{1}\right) \ldots \varphi_{\mu_{n}}\left(f_{n}\right)
$$

$$
\begin{gathered}
=E\left\{\sum_{\mu_{1}^{\prime} \ldots \mu_{n}^{\prime}} A_{\mu_{1} \mu_{1}^{\prime}}^{-1} \ldots A_{\mu_{n} \mu_{n}^{\prime}}^{-1} \varphi_{\mu_{1}^{\prime}}\left(f_{1 \mathscr{A}}\right) \ldots \varphi_{\mu_{n}^{\prime}}\left(f_{n \mathscr{A}}\right)\right\} \\
f_{k \mathscr{A}}(x)=f_{k}\left(A^{-1}(x-a)\right), \quad k=1, \ldots n .
\end{gathered}
$$

A Gaussian vector random field of mean zero is Euclidean if the general covariance of any two components of any vector random variable(s) satisfies

$$
E \varphi_{\mu_{1}}\left(f_{1}\right) \varphi_{\mu_{2}}\left(f_{2}\right)=E\left\{\sum_{\mu_{1}^{\prime} \mu_{2}^{\prime}} A_{\mu_{1} \mu_{1}^{\prime}}^{-1} A_{\mu_{2} \mu_{2}^{\prime}}^{-1} \varphi_{\mu_{1}^{\prime}}\left(f_{1 \mathscr{A}}\right) \varphi_{\mu_{2}^{\prime}}\left(f_{2 \mathscr{A}}\right)\right\} .
$$

We now define a Markov random field over $\mathscr{D}_{r}\left(R^{4}\right)$. We follow closely Nelson's definition for scalar fields. We let $U$ be an open subset of $R^{4}$, and define $\sigma(U)$ to be the $\sigma$-algebra generated by all components of the vector random variables $\Phi(f)$ with $f \in \mathscr{D}_{r}\left(R^{4}\right)$ and supp $f \subset U$. For a closed set $V$ we let $\sigma(V)=\cap \sigma(U)$ with the intersection taken over all open sets $U$ containing $V$. Then a vector random field is called Markov if

$$
E\left\{u \mid \sigma\left(U^{\prime}\right)\right\}=E\{u \mid \sigma(\partial U)\}
$$

for all positive random variables $u$ measurable with respect to $\sigma(U)$, where $U^{\prime}$ is the complement of $U$ and $\partial U$ the boundary of $U$.

The Real Proca Wightman Field is a free vector Wightman field with the following two point function:

$$
W_{\mu v}(x-y)=-\left(g^{\mu \nu}+\frac{1}{m^{2}} \partial_{\mu} \partial_{v}\right) W(x-y)
$$

where $W(x-y)$ is the two point Wightman function of the Free Scalar Wightman Field and $g^{\mu \nu}=g^{\mu} \delta_{\mu \nu}, g^{0}=1, g^{1}=g^{2}=g^{3}=-1$.

\section{The Field and Proof of the Result}

The field $\Phi(f)$ which we mentioned in Section I is a Gaussian vector random field over $\mathscr{D}_{r}\left(R^{4}\right)$ with mean zero and general covariance given by

$$
E \varphi_{\mu}(f) \varphi_{\nu}(g)=\left\langle f, \frac{\delta_{\mu \nu}-\frac{1}{m^{2}} \partial_{\mu} \partial_{v} g}{-\Delta+m^{2}}\right\rangle_{L^{2}}, \quad \mu, v=0,1,2,3,
$$


where $\Delta$ is the 4-dimensional Laplacian operator and $\langle,\rangle_{L^{2}}$ is the scalar product in $L^{2}\left(R^{4}\right)$. We now proceed to prove the result.

First we note that the vector random field is Euclidean from (3.1) and that (1.1) is true by direct computation. (1.2) is true using Wick's Theorem for jointly Gaussian random variables. We proceed to prove the Markov property of the field.

In order to do so, we first construct a Hilbert space as follows.

We let $F$ be the set $\left\{f_{0}, f_{1}, f_{2}, f_{3}\right\}$ of functions each belonging to $\mathscr{D}_{r}\left(R^{4}\right)$. So $F \in \mathscr{D}_{r}\left(R^{4}\right) \times \mathscr{D}_{r}\left(R^{4}\right) \times \mathscr{D}_{r}\left(R^{4}\right) \times \mathscr{D}_{r}\left(R^{4}\right)=\mathscr{D}_{4 r}\left(R^{4}\right)$. We introduce an inner product on $\widetilde{\mathscr{D}_{4}}\left(R^{4}\right)$, the Fourier Transform space of $\mathscr{D}_{4 r}\left(R^{4}\right)$, by

$$
\{\tilde{F}, \tilde{G}\}_{\mathscr{L}^{2}}=\sum_{\mu=0}^{3}\left\langle\tilde{f_{\mu}}, \tilde{g}_{\mu}\right\rangle_{L^{2}}
$$

where the symbol denotes the Fourier Transform. We also introduce the following inner product on $\mathscr{D}_{4 r}\left(R^{4}\right)$, analogously,

$$
\langle F, G\rangle_{\mathscr{L}^{2}}=\sum_{\mu=0}^{3}\left\langle f_{\mu}, g_{\mu}\right\rangle_{L^{2}} .
$$

We next define the following inner product on $\mathscr{D}_{4 r}\left(R^{4}\right)$ :

$$
\langle F, G\rangle=\{\tilde{F}, \tilde{K} \tilde{G}\}_{\mathscr{L}^{2}}
$$

where $\tilde{K}$ is defined by

with

$$
(\tilde{K} \tilde{G})_{\mu}(p)=\sum_{\nu=0}^{3} \tilde{K}_{\mu \nu}(p) \tilde{g}_{\nu}(p), \quad \mu=0,1,2,3
$$

$$
\tilde{K}_{\mu \nu}(p)=\frac{\delta_{\mu \nu}+\frac{1}{m^{2}} p_{\mu} p_{\nu}}{p^{2}+m^{2}} .
$$

We let $\mathscr{H}^{\prime}$ denote the pre-Hilbert space $\mathscr{D}_{4 r}\left(R^{4}\right)$ equipped with the scalar product $\langle$,$\rangle , and we let \mathscr{H}$ be the completion of $\mathscr{H}^{\prime}$ with respect to the norm defined by the scalar product $\langle$,$\rangle . We now define a map \varphi$ from $\mathscr{H}$ to $\mathscr{L}^{2}(\Omega, \mathscr{B}, \mu)$. For $F \in \mathscr{D}_{4 r}\left(R^{4}\right)$, we define

$$
\varphi(F)=\varphi_{0}\left(f_{0}\right)+\varphi_{1}\left(f_{1}\right)+\varphi_{2}\left(f_{2}\right)+\varphi_{3}\left(f_{3}\right) .
$$

Let $\mathscr{F} \in \mathscr{H}$, so that $\mathscr{F}$ is a fundamental sequence $\left\{F_{1}, F_{2}, \ldots\right\}, F_{j} \in \mathscr{H}^{\prime}$. We define $\varphi(\mathscr{F})$ to be the fundamental sequence $\left\{\varphi\left(F_{1}\right), \varphi\left(F_{2}\right), \ldots\right\} \in \mathscr{L}^{2}(\Omega, \mathscr{B}, \mu)$. We have

$$
E \varphi(\mathscr{F}) \varphi(\mathscr{G})=\langle\mathscr{F}, \mathscr{G}\rangle
$$

for $\mathscr{F}$ and $\mathscr{G}$ in $\mathscr{H} . \varphi$ is then a Gaussian random process [2] on $\mathscr{H}$ if we assume that $\mathscr{B}$ is the $\sigma$-algebra generated by all $\varphi_{\mu}(f), f \in \mathscr{D}_{r}\left(R^{4}\right), \mu=0,1,2,3$.

We now introduce the spaces

$$
\begin{aligned}
M & =\left\{\mathscr{F} \in \mathscr{H}: \operatorname{supp} \mathscr{F} \subset U^{\prime}\right\} \\
N & =\{\mathscr{F} \in \mathscr{H}: \operatorname{supp} \mathscr{F} \subset \partial U\} .
\end{aligned}
$$


We let $\mathscr{F}$ be an element of $\mathscr{H}$ with supp $\mathscr{F} \subset U$. We show that the orthogonal projection $\mathscr{F}_{M}$ of $\mathscr{F}$ onto $M$ is in $N$. To see this, we let $G \in \mathscr{D}_{4 r}\left(U^{\prime}\right)$, where $U^{\prime 0}$ is the interior of $U^{\prime}$. Then $G$ belongs to $M$. Hence we have

$$
\left\langle G, \mathscr{F}_{M}\right\rangle=\langle G, \mathscr{F}\rangle .
$$

We also have

$$
\begin{aligned}
\left\langle G, \mathscr{F}_{M}\right\rangle & =\int d^{4} p \sum_{\mu=0}^{3} \tilde{G}_{\mu}(p)^{*}\left(\tilde{K} \tilde{\mathscr{F}}_{M}\right)_{\mu}(p) \\
& \left.=\int d^{4} p \widetilde{G(p)}\right)^{+} \frac{1}{\tilde{Q}(p)} \tilde{\mathscr{F}}_{M}(p)
\end{aligned}
$$

where $\tilde{G}(p)$ and $\tilde{\mathscr{F}}_{M}(p)$ are regarded as column vectors and $\tilde{Q}(p)$ is a real $4 \times 4$ non-singular matrix whose elements are polynomials in $p_{\mu} p_{v}, \mu, v=0,1,2,3$. This follows from the crucial fact that the determinant of the matrix whose elements are $(\underset{v}{K}(p))_{\mu \nu}=\delta_{\mu v}+\frac{p_{\mu} p_{v}}{m^{2}}$ is $C\left(p^{2}+m^{2}\right)$, where $C>0$.

Since $G \in \mathscr{D}_{4 r}\left(U^{\prime}\right)$, all components of the column vector $(\widetilde{\tilde{Q} \tilde{G}})(x)$ belongs to $\mathscr{D}_{r}\left(U^{\prime}{ }^{0}\right)$. Hence

$$
\left\langle Q G, \mathscr{F}_{M}\right\rangle=\langle Q G, \mathscr{F}\rangle
$$

where $Q$ is a real $4 \times 4$ matrix differential operator whose Fourier Transform is the multiplicative matrix operator $\tilde{Q}(p)$. We have

$$
\begin{aligned}
\left\langle Q G, \mathscr{F}_{M}\right\rangle & =\int d^{4} p(\widetilde{Q G})^{+}(p) \frac{1}{\tilde{Q}(p)} \tilde{\mathscr{F}}_{M}(p) \\
& =\int d^{4} p \tilde{G}^{+}(p) \tilde{Q}(p) \frac{1}{\tilde{Q}(p)} \tilde{\mathscr{F}}_{M}(p)=\left\{\tilde{G}, \tilde{\mathscr{F}}_{M}\right\}_{\mathscr{L}^{2}} \\
\langle Q G, \mathscr{\mathscr { F }}\rangle & =\{\tilde{G}, \tilde{\mathscr{F}}\}_{\mathscr{L}^{2}} .
\end{aligned}
$$

Hence we have

$$
\left\langle G, \mathscr{F}_{M}\right\rangle_{\mathscr{L}^{2}}=\langle G, \mathscr{F}\rangle_{\mathscr{L}^{2}}=0 .
$$

We therefore obtain $\mathscr{F}_{M} \in N$.

Hence, following Nelson [3], we have the Markov property of the field $\Phi$ over $\mathscr{D}_{r}\left(R^{4}\right)$ defined above, noting that the $\sigma$-algebra generated by $\left\{\varphi(F), F \in \mathscr{D}_{4 r}(\Lambda)\right\}$ is the same as the $\sigma$-algebra generated by

$$
\left\{\varphi_{\mu}(f), f \in \mathscr{D}_{r}(\Lambda), \mu=0,1,2,3\right\},
$$

for any open set $\Lambda$ in $R^{4}$.

Acknowledgments. The author would like to thank Professor R. F. Streater and Dr. M. Hasler for helpful discussions, and G. Ekhaguere and S. C. Lim for conversations. He also wishes to thank Bedford College for hospitality and the Science Research Council for financial support. 


\title{
References
}

1. Gross, L.: The free euclidean proca and electromagnetic fields. Cumberland Lodge Conference Report, 1974

2. Simon, B.: The $P(\phi)_{2}$ euclidean (quantum) field theory. Princeton University Press 1974

3. Velo, G., Wightman, A., (Ed.): Constructive quantum field theory, pp. 106-107. Berlin-HeidelbergNew York: Springer 1973

Communicated by A. S. Wightman

\author{
T. H. Yao \\ Mathematics Department \\ Bedford College \\ Regent's Park \\ London NW1, England
}


